\title{
Allozyme analysis of Haemonchus contortus resistant and susceptible to anthelmintics, with an indication of dipeptidases associated with resistance
}

\author{
V. ŠNÁBEL ${ }^{1 *}$, A. KÖNIGOVÁ ${ }^{1}$, M. DOLINSKÁ ${ }^{1}$, A. J. WOLSTENHOLME ${ }^{2,3}$, M. VÁRADY ${ }^{1}$ \\ ${ }^{1}$ Institute of Parasitology, Slovak Academy of Sciences, Hlinkova 3, 04001 Košice, Slovakia, \\ *E-mail: snabel@saske.sk; ${ }^{2}$ University of Bath, Department of Biology and Biochemistry, Bath BA2 7AY, U.K.; \\ ${ }^{3}$ Department of Infectious Diseases and Center for Tropical and Emerging Global Diseases, University of Georgia, \\ Athens, GA 30602, USA
}

\begin{abstract}
Summary
In an attempt to investigate the genetic and biochemical causes underlying resistance to anthelmintics, the trichostrongylid nematode Haemonchus contortus was examined by an allozymic analysis of nine candidate gene-enzyme systems. Two strains resistant to benzimidazoles, two multidrug-resistant strains, and three strains susceptible to anthelmintics isolated from three continents were examined by isoelectric focusing. Two enzymes (mannose phosphate isomerase and peptidase-D) had polymorphic patterns. The peptidase-D zymograms indicated an association with the selection for resistance. Susceptible isolates gave more complex patterns due to the formation of a heterodimer controlled by the two active loci, whereas in resistant isolates, only a single locus with one electromorph was responsible for the profiles. A possible link between dipeptidases and the acquisition of resistance through the turnover of protein-rich collagen, and changes in membrane-associated genes that influence drug efflux, are discussed.
\end{abstract}

Keywords: Haemonchus; resistance; peptidase; strain

\section{Introduction}

Haemonchus contortus, a common trichostrongylid nematode of sheep and goats, is responsible for outbreaks of haemonchosis associated with sudden mortalities, particularly in young animals in the subtropics and tropics. Haemonchosis is also an economically important disease in temperate climates where it negatively affects productivity and the welfare of livestock (Eysker et al., 2005). Due to the widespread use of anthelmintics, gastro-intestinal parasites of small ruminants and particularly $H$. contortus have developed resistance to the major classes of drugs used for its control (Várady et al., 2011). Research aimed at identifying molecular markers for anthelmintic resistance would assist in our understanding of the pro- cesses involved in resistance and provide tools for screening for resistance. The genetic and biochemical mechanisms underlying resistance are not satisfactorily understood but are complex and appear to vary among different species of helminths (Beech et al., 2011). Physiological homeostasis, through the establishment of alternative metabolic pathways, represents a frequent mechanism of resistance, which appears to develop more rapidly when the target of a drug is an enzyme rather than an ion channel (Hennessy, 1999; Köhler, 2001). For example, the observed resistance in Schistosoma mansoni against oxamniquine and structurally-related hycanthone is due to the absence of an enzyme activity mediating the covalent binding of these drugs to DNA in resistant isolates (PicaMattoccia et al., 1997). Multilocus enzyme electrophoresis is a useful technique for studying selected gene-enzyme systems in parasitic populations (Andrews \& Chilton, 1999). This approach has only rarely been employed in studies of genetic mechanisms of resistance in parasitic helminths. In an allozymic study of $H$. contortus strains originating from Australia, UK and South Africa, Sutherland et al. (1988) found a significantly greater esterase activity and pattern complexity in benzimidazole (BZ) resistant (seven bands) than in sensitive (two bands) isolates with the isoelectric focusing technique employed. Using starch gel electrophoresis, Echevarria et al. (1992) found differences in propionyl esterases between ivermectin-resistant and ivermectin-sensitive strains of $H$. contortus from the UK and South Africa. The authors proposed that the loss of propionyl esterase activity was associated with ivermectin resistance, and in a multidrug-resistant strain this loss of activity was masked by BZ resistance. In another strongylid nematode, Oesophagostomum dentatum, significant differences in allelic frequencies of mannose phosphate isomerase ( $\mathrm{Mpi}$ locus) were found by isoelectric focusing between strains sensitive and resistant to drugs of the imidothiazole/tetrahydropyrimidine anthel- 
Table 1. Haemonchus contortus strains subjected to allozymic analyses

\begin{tabular}{lll}
\hline Strain code & Resistance/susceptibility & Origin/source \\
\hline MHco13 & BZ res. & Switzerland/Novartis Centre de Recherche Santé Animale (St. Aubin, Switzerland) \\
MHco12 & BZ res. & Switzerland/Novartis Centre de Recherche Santé Animale (St. Aubin, Switzerland) \\
MHco4 (WRS) & IVM/BZ/RAF/CLOS res. & South Africa/Moredun Research Institute (Edinburgh, UK) \\
MHco10 (CAVR) & IVM/BZ res. & Australia/CSIRO Pastoral Research Laboratory (Armidale, Australia) \\
MHco3 (ISE) & Susceptible & Kenya/Moredun Research Institute (Edinburgh, UK) \\
MHco9 & Susceptible & Germany/Bayer HealthCare AG (Hannover, Germany) \\
MHco6 & Susceptible & Kenya/Novartis Centre de Recherche Santé Animale (St. Aubin, Switzerland) \\
\hline
\end{tabular}

mintic group (Šnábel et al., 2000). The mannose-6-phosphate receptor is a membrane-bound glycoprotein that mediates the sorting of lysosomal enzymes in phosphorylated mannose residues (Nicoziani et al., 2000). Šnábel et al. (2000) suggested that changes in the allelic composition of the Mpi locus might imply alterations in the function of the receptor. The purpose of the present study was to characterise qualitative allozymic changes in selected strains of $H$. contortus that occur as resistance develops.

\section{Material and methods}

Four anthelmintic-resistant and three susceptible strains of $H$. contortus were used for the electrophoretic experiments (see Table 1). The BZ-resistant isolates MHco13 and MHco12 were recently isolated from Swiss farms. The MHco4 (the White River strain, WRS) strain was originally isolated from the Lowveld of the Transvaal in South Africa as a field isolate resistant to BZs, ivermectin (IVM), and the salicylanilide anthelmintics closantel (CLOS) and rafoxanide (RAF) (Van Wyk \& Malan, 1988). MHco4 is now maintained at the Moredun Research Institute (Edinburgh, UK) as a multidrug-drug resistant strain. MHco10 (CAVR) arose as a serendipitous ivermectinresistant contaminant of a passaged laboratory strain of Trichostrongylus colubriformis in Armidale, New South Wales, Australia (Le Jambre, 1993). This isolate was subsequently characterised as resistant to ivermectin and BZs but susceptible to levamisole and salicylanilide anthelmintics (Le Jambre et al., 1995). The susceptible strain MHco3 (ISE) was inbred from a highly heterogeneous outbred population that had been maintained at the Moredun Research Institute since the 1950s but which may have originated in Kenya (Otsen et al., 2001). MHco9 and MHco6 were originally isolated as susceptible isolates of sheep from Germany and Kenya, respectively.

For electrophoretic analyses, individual adult worms of both sexes were crushed in an equal volume of distilled water. Homogenates were centrifuged for $10 \mathrm{~min}$ at 12000 $\mathrm{g}$, and supernatants were analysed by isoelectric focusing (IEF) using the Multiphor II Electrophoresis System equipped with a MultiTemp III Thermostatic Circulator (Amersham Biosciences). Enzyme extracts were applied in a volume of $15 \mu \mathrm{l}$ onto Ampholine PAGplates (Amersham Biosciences) with $\mathrm{pH}$ ranges of $3.5-9.5$ and $4.0-6.5$, respectively, and subjected to electrophoresis for $1.5 \mathrm{~h}$ under conditions described in Hanzelová et al. (1999). After electrophoresis, gels were stained for the chosen enzymes using recipes modified from Murphy et al. (1996), and developing solutions were applied in the $1.2 \%$ agar overlay. Zymograms with a sufficient intensity of staining were obtained for the following enzymes: adenylate kinase (AK, EC 2.7.4.3), fumarate hydratase (FUMH, EC 4.2.1.2), glutamate dehydrogenase (GLD, EC 1.4.1.2), glycerophosphate dehydrogenase (GPD, EC 1.1.1.8), glucose phosphate isomerase (GPI), mannose phosphate isomerase (MPI), peptidase B with leucine-leucine-leucine substrate (PEP-B, EC 3.4.11.1), peptidase $\mathrm{D}$ with phenylalanine-proline substrate (PEP-D, EC 3.4.13.9), and phosphoglucomutase (PGM, EC 5.4.2.2). Presumed allozymes and isoenzymes were numbered in increasing order of electrophoretic mobility from the cathode relative to the most common signal (which was assigned a mobility value of 100 ).

Fisher's exact t-test was used to assess the statistical significance of differences in genotypic and allelic frequencies between strains under R (R Development Core Team, 2010). Cluster analysis of similarity between electrophoretic patterns with resulting dendrograms was derived by the UPGMA method using MEGA5 software (Tamura et al., 2011).

\section{Results}

Five of the nine candidate enzymes tested by IEF (AK, FUMH, GLD, GPD, PEP-B) exhibited one major consistent zone of activity. Identical profiles among strains were also recorded with PGM, in which the main signal was accompanied by one cathodal and two anodal bands, and in GPI that generated an intense central band with one cathodal and four anodal bands. Two enzymes (MPI and PEP-D) had polymorphic and variable electrophoretic patterns. The variability of MPI was interpretable in a genetic context, allowing us to derive an allelic profile for each sample. Two alleles corresponding to monomeric polypeptides were screened. A major allele (100), with greater electrophoretic mobility, associated in most samples with a 100/100 homozygous genotype, and a rare allele (90), with frequencies ranging from 0.07 to 0.17 in individual strains, were observed (genotypic and allelic frequencies of MPI are given in Table 2). Of the 51 sam- 
Table 2. Genotypes and allelic frequencies of MPI

\begin{tabular}{lcccccc}
\hline Strain (N) & Res./susc. & \multicolumn{3}{c}{$\begin{array}{c}\text { Number of observed } \\
\text { genotypes }\end{array}$} & \multicolumn{2}{c}{ Allelic frequencies } \\
& & $\mathbf{9 0 / 9 0}$ & $\mathbf{9 0 / 1 0 0}$ & $\mathbf{1 0 0 / 1 0 0}$ & $\mathbf{9 0}$ & $\mathbf{1 0 0}$ \\
\hline & & & & & & \\
MHco13 (9) & res. & 1 & 1 & 7 & 0.17 & 0.83 \\
MHco12 (6) & res. & - & 1 & 5 & 0.08 & 0.92 \\
MHco4 (8) & res. & 1 & - & 7 & 0.13 & 0.87 \\
MHco10 (7) & res. & 1 & - & 6 & 0.14 & 0.86 \\
MHco3 (8) & susc. & 1 & - & 7 & 0.13 & 0.87 \\
MHco9 (6) & susc. & - & 2 & 4 & 0.17 & 0.83 \\
MHco6 (7) & susc. & - & 1 & 6 & 0.07 & 0.93 \\
\hline N - number of examined specimens of $H$. contortus strains &
\end{tabular}

ples examined for MPI, three 90/100 heterozygotes were identified in the susceptible isolates and two in the resistant isolates. The overall frequencies of alleles 100 and 90 were $88.1 \%$ and $11.9 \%$ in the susceptible strains, and $86.7 \%$ and $13.3 \%$ in the resistant strains, respectively. Fisher's exact t-test did not indicate any significant differences in genotypic and allelic frequencies of MPI between the resistant and susceptible strains $(P$ values were 0.65 and 0.83 , respectively).

Table 3. Frequencies of isoenzyme records in strain zymograms of PEP-D (\%)

\begin{tabular}{lccc}
\hline Strain (N*) & $\begin{array}{c}\text { Isoenzyme } \\
80\end{array}$ & $\begin{array}{c}\text { Isoenzyme } \\
90\end{array}$ & $\begin{array}{c}\text { Isoenzyme } \\
100\end{array}$ \\
\hline MHco13 (6) & - & 16.7 & 100 \\
MHco12 (7) & - & - & 100 \\
MHco4 (6) & - & - & 100 \\
MHco10 (5) & - & - & 100 \\
MHco3 (6) & 100 & 100 & 100 \\
MHco9 (6) & 33.3 & 50 & 100 \\
MHco6 (5) & 100 & 100 & 100 \\
*N - number of examined specimens of H. contortus strains
\end{tabular}

The electrophoretic profiles for PEP-D showed striking differences between the two groups of strains. The frequencies of the isoenzymes detected in the zymograms for each strain are given in Table 3 . The pattern of variability was more complex in the susceptible isolates. Given relatively large distances among three recorded electromorphs and proportions of band intensities, the most plausible interpretation for the patterns obtained was their generation by the hybridization of two loci (Pep-D-1, Pep-D-2) forming heterodimers of the isoenzymes with mobilities of 80, 90, and 100 (Fig. 1, lanes $8-11$ ), rather than by allozymes attributed to a single locus. In contrast, the resistant isolates expressed only one active locus ( $P e p-D$ 1 ), with the isoenzyme having mobility 100 (shared with susceptible isolates). This electromorph was detected in 23 resistant worms. One resistant worm from strain MHco13 (BZ resistant) also manifested isoenzyme 90 (Fig. 1, lane 2). All 11 samples of the susceptible Kenyan isolates

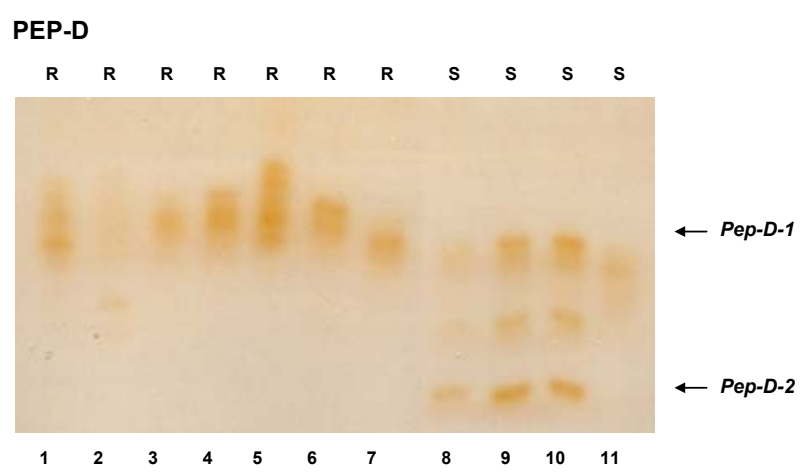

Fig. 1. Zymogram of peptidase D (PEP-D) obtained by isoelectric focusing on polyacrylamide gels. Lane 1, MHco13 (isoenzyme 100); lane 2, MHco 13 (isoenzymes 90, 100); lane 3, MHco13 (isoenzyme 100); lane 4, MHco12 (isoenzyme 100); lane 5, MHco12 (isoenzyme 100); lane 6, MHco12 (isoenzyme 100); lane 7, MHco4 (isoenzyme 100); lane 8, MHco3 (isoenzymes 80, 90, 100); lane 9, MHco3 (isoenzymes 80, 90, 100); lane 10, MHco6 (isoenzymes 80, 90, 100); lane 11, MHco9 (isoenzyme 100). R, resistant strain; $\mathrm{S}$, susceptible strain

(MHco3 and MHco6) had isoenzymes 80, 90, and 100. In MHco9 from Germany, isoenzyme 100 occurred in all samples, and two additional signals were alternating: isoenzyme 90 was present in three of six worms and isoenzyme 80 in two of six worms (Table 3). A UPGMA analysis of the PEP-D polymorphisms (presence/absence of electromorph) corroborated the divergence between the profiles of resistant and susceptible strains of $H$. contortus, with the two groups forming separate clusters (Fig. 2).

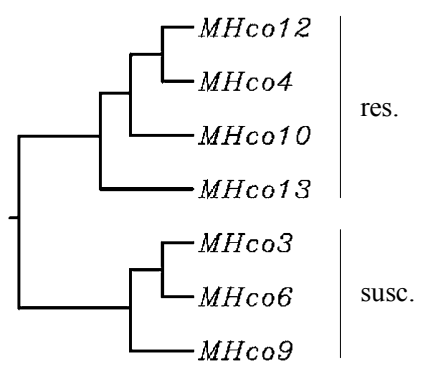

Fig. 2. Dendrogram derived from PEP-D patterns of $H$. contortus strains obtained by UPGMA analysis 


\section{Discussion}

Trichostrongylid nematodes are characterised by a high degree of genetic variability, which is primarily influenced by their large effective population sizes (Anderson et al., 1998; Grillo et al., 2007). Beech et al. (1994) measured a nucleotide diversity of $0.091-0.094$ in isotypes 1 and 2 of $\beta$-tubulin in $H$. contortus, which was higher than the diversity in nuclear introns $(0.02-0.08)$ of ascarid nematodes from smaller populations (Anderson \& Jaenike, 1997). In an analysis of the mitochondrial nad4 gene from an alpine population of $H$. contortus (Cerutti et al., 2010), the average nucleotide diversity between individuals was $4 \%$. This was a higher value than that estimated by Blouin et al. (1997) for a U.S. population (2.6\%) and is consistent with the hypothesis that the European population is the ancestral one. In our allozyme study of polymorphisms in $H$. contortus and their possible link with anthelmintic resistance, we studied gene-enzyme systems that are not part of key metabolic pathways and are more amenable to adapt to selection pressure ( 6 enzymes), enzymes that might influence the binding of drugs to glutamate-gated chloride channels (GLD, GPD), and enzymes mediating pathways associated with the action or synthesis of P-glycoproteins acting as membrane-bound transporters (MPI). Of the nine candidate enzymes that provided good resolution, two displayed polymorphic and variable electrophoretic patterns in the seven strains examined. PEP-D showed obvious differences between the resistant and susceptible isolates, as seen in the clustering pattern of Fig. 2. The more complex banding patterns controlled by two loci in the susceptible isolates (unlike the single locus expressed in the resistant isolates) seen in examined $H$. contortus strains suggest a scenario in the development of resistance similar to that presented by Kwa et al. (1993) who showed that the $\beta$-tubulin isotype 2 gene may be deleted in highly BZresistant strains. The question arises whether the variation in the PEP-D profiles is due to the acquisition of resistance or to the different geographical origins of the strains. Two susceptible strains (MHco3 and MHco6) originated in Kenya, whereas the other (MHco9) was isolated in Germany. The marked genetic divergence of Kenyan populations of $H$. contortus was demonstrated in the population study of Troell et al. (2006), in which isolates from Africa (Kenya and Ethiopia but excluding the resistant WSE strain from South Africa, also analyzed here) showed almost no structuring within mitochondrial nad4 sequences and AFLP data and differed substantially from populations from other continents. Nevertheless, the German MHco9 susceptible strain and the two Kenyan susceptible strains had isoenzymes 80 and 90 that were absent (except for one sample) in the resistant strains. Differences in the PEP-D profiles are, therefore, more likely to be attributed to drug selection rather than to geographical origin or to other genetic processes such as inbreeding or bottlenecks during laboratory passages. The detection of isoenzyme 90 in one sample of the resistant MHco13 strain from Switzerland may indicate that a minor proportion of susceptible alleles can also be present in resistant lines.

PEP-D belongs to the dipeptidase class of enzymes that hydrolyze single amino acids from small peptides. Peptidases are being extensively studied in nematodes from various physiological aspects, e.g. in providing a better understanding of key reproductive processes, antigen functioning in connection with vaccine development, and processes associated with the migration of embryonic muscle cells (Yatsuda et al., 2003; Geldhof and Knox, 2008; Li et al., 2011). That peptidases act similarly in related nematode organisms was documented in a transcriptomic analysis by Cantacessi et al. (2010), who inferred that the 211 predicted peptides of the strongylid parasite Trichostrongylus colubriformus assigned to biological pathways linked to peptidases had significant homology to a total of 38 unique peptidases of the free-living nematode Caenorhabditis elegans. Laurent et al. (2001) detected a strong expression of peptidases in the intestinal cells of $C$. elegans, suggesting that these enzymes are primarily involved in the intracellular hydrolysis of proline-containing peptides generated during digestion of bacteria in the lumen of the intestine. More importantly in relation to anthelmintic resistance, peptidases function in the turnover of proline-rich collagen. Collagen is used extensively in nematodes for the periodic synthesis of their protective cuticle during moulting, and re-cycling of the cuticular components requires peptidases capable of degrading proline-rich proteins (Bird \& Bird, 1991). This fibrous protein is also a key component of the basement membranes in nematodes, and peptidases are therefore likely to be involved in the hydrolysis of collagen-derived peptides. The observed differences in the PEP-D electrophoretic patterns may be associated with altered membrane-bound proteins that play an important role in drug transport and efflux from cells and may thus influence the development of resistance in $H$. contortus.

No striking differences were found between the PEP-D profiles of the resistant strains despite the fact that the respective strains were demonstrably resistant to a variety of anthelmintics; MHcol3 and MHco12 were selected with BZs, while MHco10 (CAVR) and MHco4 (WRS) are multiply resistant to two (BZs and macrocyclic lactones) and three (BZs, macrocyclic lactones, and salicylanilides) anthelmintic classes, respectively. Thus, a more generic, non-specific resistance mechanism associated with dipeptidases may be acting in these organisms. In recent studies, altered allelic frequencies of $\beta$-tubulin genes were found in populations of $H$. contortus resistant to macrocyclic lactones that indicate a possible genetic link between BZ resistance and macrocyclic lactone resistance (Eng et al., 2006; de Lourdes Mottier and Prichard, 2008). It is not yet clear if changes in $\beta$-tubulin proteins can also induce resistance to macrocyclic lactones or if the changes are caused by genetic hitchhiking (Beech et al., 2011).

Most of the research into genetic and biochemical markers for resistance has focused on the possible associations 
between candidate genes and resistance phenotypes. The present study complements the earlier reports of Sutherland et al. (1988) and Echevarria et al. (1992) indicating allozymic changes that accompany the development of anthelmintic resistance in $H$. contortus. The nature of the variation associated with dipeptidase expression merits further investigation through the sequencing of the genes encoding these peptidases, followed by a detailed allele frequency analysis in Haemonchus strains of known resistance status in order to identify domains putatively associated with resistance.

\section{Acknowledgements}

The work was supported by the EU FP6 PARASOL project (FOOD-CT-2005022851), grants of the Scientific Grant Agency VEGA (projects Nos. 2/0213/10, $2 / 0135 / 10)$, and by the Slovak Research and Development Agency (project No. LPP-0186-0).

\section{References}

Anderson, T. J. C., JAENIKE, J. (1997): Host specificity, evolutionary relationships and macrogeographic differentiation among Ascaris populations from humans and pigs. Parasitology, 115(Part 3): 325 - 342. DOI: 10.1017/S0031182097001339

Anderson, T. J., Blouin, M. S., BeEch, R. N. (1998): Population biology of parasitic nematodes: applications of genetic markers. Adv. Parasitol., 41: 219 - 283. DOI: 10.1016/S0065-308X(08)60425-X

Andrews, R. H., Chilton, N. B. (1999): Multilocus enzyme electrophoresis: a valuable technique for providing answers to problems in parasite systematics. Int. J. Parasitol., 29(2): 213 - 253. DOI: 10.1016/S0020-7519(98) 00168-4

Beech, R. N., Skuce, P., Bartley, D. J., Martin, R. J., PRICHARD, R. K., GILLEARD, J. S. (2011): Anthelmintic resistance: markers for resistance, or susceptibility? Parasitology, 138(2): 160 - 174. DOI: 10.1017/S0031182010001198

BIRD, A. F., BIRD, J. (1992): The structure of nematodes, San Diego, California, Academic Press, 315 pp.

Blouin, M. S., Yowell, C. A., Courtney, C. H., DAme, J. B. (1997): Haemonchus placei and Haemonchus contortus are distinct species based on mtDNA evidence. Int. J. Parasitol., 27(1): 1383 - 1387. DOI: 10.1016/S00207519(97)00125-2

Cantacessi, C., Mitreva, M., Campbell, B. E., Hall, R. S., Young, N. D., JeX, A. R., Ranganathan, S., GASSER, R. B. (2010): First transcriptomic analysis of the economically important parasitic nematode, Trichostrongylus colubriformis, using a next-generation sequencing approach. Infect. Genet. Evol., 10(8): 1199 - 1207. DOI: 10.1016/j.meegid.2010.07.024

Cerutti, M. C., CitTerio, C. V., Bazzocchi, C., Epis, S., D’Amelio, S., FERRARI, N., LANFRANCHI P. (2010): Genetic variability of Haemonchus contortus (Nematoda: Trichos- trongyloidea) in alpine ruminant host species. J. Helminthol., 84(3): 276 - 283. DOI: 10.1017/S0022149X09990587

DE Lourdes MotTier, M., Prichard, R. K. (2008): Genetic analysis of a relationship between macrocyclic lactone and benzimidazole anthelmintic selection on Haemonchus contortus. Pharmacogenet. Genom., 2008, 18(2): 129 140. DOI: 10.1097/FPC.0b013e3282f4711d

ECheVArRia, F. A., GenNARI, S. M., TAIT, A. (1992): Isoenzyme analysis of Haemonchus contortus resistant or susceptible to ivermectin. Vet. Parasitol., 44(1 - 2): 87 95. DOI: 10.1016/0304-4017(92)90146-Z

Eng, J. K., Blackhall, W. J., Osei-Atweneboana, M. Y., Bourguinat, C., Galazzo, D., Beech, R. N., Unnasch, T. R., Awadzi, K., Lubega, G. W., Prichard, R. K. (2006): Ivermectin selection on beta-tubulin: evidence in Onchocerca volvulus and Haemonchus contortus. Mol. Biochem. Parasitol., 150(2): 229 - 235. DOI: 10.1016/j.molbiopara.2006.08.007

Eysker, M., BAKKer, N., KoOyman, F. N., Ploeger, H. W. (2005): The possibilities and limitations of evasive grazing as a control measure for parasitic gastroenteritis in small ruminants in temperate climates. Vet. Parasitol., 129(1 - 2): 95 - 104. DOI: 10.1016/j.vetpar.2004.12.022

Geldhof, P., KNOX, D. (2008): The intestinal contortin structure in Haemonchus contortus: An immobilised anticoagulant? Int. J. Parasitol., 38(13): 1579 - 1588. DOI: 10.1016/j.ijpara.2008.05.002

GRANT, W. N. (1994): Genetic variation in parasitic nematodes and its implications. Int. J. Parasitol., 24(6): 821 830. DOI: 10.1016/0020-7519(94)90008-6

Grillo, V., JaCkson, F., CABARET, J., Gilleard, J. S. (2007): Population genetic analysis of the ovine parasitic nematode Teladorsagia circumcincta and evidence for a cryptic species. Int. J. Parasitol., 37(3-4): 435 - 447. DOI: 10.1016/j.ijpara.2006.11.014

HANZElovÁ, V., ŠNÁBel, V., KRÁlovÁ, I., Scholz, T., D'AMELIO, S. (1999): Genetic and morphological variability in cestodes of the genus Proteocephalus: geographical variation in P. percae populations. Can. J. Zool., 77(9): 1450 - 1458. DOI: $10.1139 / \mathrm{cjz}-77-9-1450$

Hennessy, D. R. (1999): Novel chemical targets. Int. J. Parasitol., 29(1): 113 - 114. DOI: 10.1016/S0020-7519 (98)00194-5

KÖHLER, P. (2001): The biochemical basis of anthelmintic action and resistance. Int. J. Parasitol., 31(4): 336 - 345. DOI: 10.1016/S0020-7519(01)00131-X

Kwa, M. S. G., Kooyman, F. J., Boersema, J. H., Roos, M. H. (1993): Effect of selection for benzimidazole resistance in Haemonchus contortus on $\beta$-tubulin isotype 1 and isotype 2 genes. Biochem. Biophys. Res. Commun., 191(2): 413 - 419. DOI: 10.1006/bbrc.1993.1233

LAURent, V., Brooks, D. R., CoATes, D., IsAaC, R. E. (2001): Functional expression and characterization of the cytoplasmic aminopeptidase P of Caenorhabditis elegans. Eur. J. Biochem., 268(20): 5430 - 5438. DOI: 10.1046/ j.0014-2956.2001.02483.x

LE JAMBRE, L. F. (1993): Ivermectin-resistant Haemonchus contortus in Australia. Aust. Vet. J., 70(9): 357. 
DOI: 10.1111/j.1751-0813.1993.tb00887.x

LE Jambre, L. F., GiLl, J. H., Lenane, I. J., LACEy, E. (1995): Characterisation of an avermectin resistant strain of Australian Haemonchus contortus. Int. J. Parasol., 25: 691 - 698. DOI: 10.1111/j.1751-0813.1993.tb00887.x

Li, B. W., Rush, A. C., Jiang, D. J., Mitreva, M., ABUBUCKER, S., WeIL, G. J. (2011): Gender-Associated Genes in Filarial Nematodes Are Important for Reproduction and Potential Intervention. PLoS Negl. Trop. Dis., 5(1), E947 - E947. DOI: 10.1371/journal.pntd.0000947 Murphy, R. W., Sites, JR. J. W., Buth, D. G., HAufler, C. H. (1996): Proteins: isozyme electrophoresis. In: HILLIS, D. M., MORITZ, C. (Eds): Molecular Systematics, 2nd ed. Sunderland, Massachusetts: Sinauer Assoc. Inc., pp. $51-120$

Nicoziani, P., Vilhardt, F., Llorente, A., Hilout, L., Courtoy, P. J., SANDVig, K., VAn Deurs, B. (2000): Role for dynamin in late endosome dynamics and trafficking of the cation-independent mannose 6-phosphate receptor. Mol. Biol. Cell., 11(2): 481 - 495

Otsen, M., Plas, M. E., Groenveld, J., Roos, M. H., LENSTRA, J. A., HOEKSTRA, R. (2000): Genetic markers for the parasitic nematode Haemonchus contortus based on intron sequences. Exp. Parasitol., 95(3): 226 - 229. DOI: 10.1006/expr.2000.4532

PicA-MatToccia, L., Novi, A., Cioli, D. (1997): Enzymatic basis for the lack of oxamniquine activity in Schistosoma haematobium infections. Parasitol. Res., 83(7), 687 - 689. DOI: $10.1007 / \mathrm{s} 004360050320$

R DEVElopMent CORE TEAM (2010): R: A language and environment for statistical computing. R Foundation for Statistical Computing, Vienna, Austria. ISBN 3-90005107-0, URL http://www.R-project.org.

Šnábel, V., De Meeûs, T., VÁrady, M., NANSEn, P.,
BJøRN, H., ČORBA, J. (2000): Sexually linked Mpi locus is presumably involved in imidothiazole resistance in Oesophagostomum dentatum parasites. Parasitol. Res., 86(6): 486 - 490. DOI: 10.1007/s004360050698

SuTHERLAND, I. A., LEE, D. L., LEWIS D. (1988): Detection of benzimidazole resistance in trichostrongylid nematodes. Parasitol. Today, 4(1): 22 - 24. DOI: 10.1016/01694758(88)90055-5

Tamura, K., Peterson, D., Peterson, N., Stecher, G., NEI, M., KUMAR, S. (2011): MEGA5: Molecular Evolutionary Genetics Analysis using Maximum Likelihood, Evolutionary Distance, and Maximum Parsimony Methods. Mol. Biol. Evol., 28(10), 2731 - 2739. DOI: 10.1093/molbev/msr121

Troell, K., Engström, A., Morrison, D. A., MAtTsson, J. G., HöGLund, J. (2006): Global patterns reveal strong population structure in Haemonchus contortus, a nematode parasite of domesticated ruminants. Int. J. Parasitol, 36(12), 1305 - 1316. DOI: 10.1016/j.ijpara.2006.06.015

VAN WYK, J. A., MALAN, F. S. (1988): Resistance of field strains of Haemonchus contortus to ivermectin, closantel, rafoxanide and benzimidazoles in South Africa. Vet. Record, 123(9), 226-228

VÁrady, M., PAPAdopoulos, E., DolinskÁ, M., KÖNIGOVÁ, A. (2011): Anthelmintic resistance in parasites of small ruminants: sheep versus goats. Helminthologia, 48(3), 137 - 144. DOI: 10.2478/s11687-011-0021-7

Yatsuda, A. P., Krijgsveld, J., Cornelissen, A. W., HECK, A. J., DE VRIES, E. (2003): Comprehensive analysis of the secreted proteins of the parasite Haemonchus contortus reveals extensive sequence variation and differential immune recognition. J. Biol. Chem. 278(19): 16941 16951. DOI: $10.1074 /$ jbc.M212453200 